\title{
A Twin Study of Ethanol Metabolism
}

\author{
N. G. Martin, ${ }^{1,3}$ J. PerI, ${ }^{2}$ J. G. Oakeshott, ${ }^{1}$ J. B. Gibson, ${ }^{1}$ G. A. \\ Starmer, ${ }^{2}$ and A. V. Wilks ${ }^{1}$
}

Received 17 July 1984-Final 20 Sept. 1984

\begin{abstract}
Blood alcohol measurements were obtained for 206 pairs of twins who had ingested a standard dose of alcohol $(0.75 \mathrm{~g} / \mathrm{kg}$ body weight) and repeat measurements were obtained for 40 of these pairs on a second occasion. The repeatability of the peak blood alcohol concentration (BAC) was 0.66 , that of the rate of elimination was 0.39 , and that of the time to peak $B A C$ was 0.27 . Only a small portion of the nonrepeatable variance could be explained by measurement error or drinking experience. It is concluded that short-term environmental factors exercise considerable influence on alcohol metabolism, particularly in the absorption phase. All of the repeatable variance in peak $B A C$ and rate of elimination was due to genetic factors. Only a small proportion of any of the genetic variance could be explained by individual differences in weight, adiposity, or lung function. Likewise, these three factors were unable to account for the fact that females had higher BACs than males during both absorption and elimination.
\end{abstract}

KEY WORDS: alcohol metabolism; twins; genetics.

\section{INTRODUCTION}

Considerable variation has been reported among individuals in the rate of elimination of ingested ethanol (e.g., Wagner, 1973) and three small twin studies have been carried out on this character. Its heritability has

This work was supported by a grant in aid from the Australian Associated Brewers.

'Department of Population Biology, Research School of Biological Sciences, Australian National University, Canberra ACT 2601, Australia.

${ }^{2}$ Department of Pharmacology, University of Sydney, Sydney NSW 2006, Australia.

${ }^{3}$ To whom correspondence should be addressed at Department of Human Genetics, Medical College of Virginia, Box 33, MCV Station, Richmond, Virginia 23298-0001. 
been estimated as 0.98 in one study involving 14 twin pairs (Vesell, 1972), 0.67 in another using 20 pairs (Luth, 1939), and 0.41 in a third using 40 pairs (Kopun and Propping, 1977). However, Wagner and Patel (1972) and Wagner (1973) have also shown that considerable variation also occurs within individuals between testing occasions. Moreover, Martin et al. (1978) showed that twin samples of the sizes used so far are too small to resolve with reasonable power the genetic and environmental contributions to individual differences. Their calculations indicate that the $95 \%$ confidence limits of the heritability estimates from the three twin studies above include both 0.0 and 1.0 .

Here we report the results of a genetical analysis of blood and breath alcohol measurements obtained in a study of 206 twin pairs who ingested a weight-related dose of ethanol. The repeatability of these measurements in 40 pairs of twins who were tested on a second occasion is also given. In a companion paper (Martin et al., 1985) we report on psychomotor performance in these twins following alcohol ingestion and on the relationships between this performance and blood alcohol levels, previous drinking experience, and other variables.

\section{SUBJECTS AND METHODS}

\section{Subjects}

Pairs of healthy twins aged between 18 and 34 years (mean, 23.1 years) were recruited from the Sydney and Canberra metropolitan areas through the Australian NH \&MRC Twin Registry. Both members of a twin pair attended on the same day and the study extended over all seasons from April 1979 to May 1981. The number of pairs tested on a given day varied between two and six and all twins were tested on either a Saturday or a Sunday. Some twins (less than 5\%) did not complete the test schedule for a variety of reasons (usually vomiting), but 206 pairs were successfully tested. Of these, 40 pairs ( 36 females and 44 males) were successfully tested again on a second occasion between 1 and 17 months (mean, 4.5 months) after the first session, in order to estimate the repeatability of the measurements. All subjects were of European (mainly northern European) extraction.

All twins were blood typed with the antisera anti-A, A, B, C, c, D, $\mathrm{E}, \mathrm{e}, \mathrm{M}, \mathrm{N}, \mathrm{S}, \mathrm{s}, \mathrm{Fy}^{\mathrm{a}}, \mathrm{K}$, and $\mathrm{Jk}^{\mathrm{a}}$ and were also typed for the serum enzyme $\alpha$-1-antitrypsin (Pi). Pairs were diagnosed as dizygotic (DZ) on the basis of a difference in sex, a difference in at least one marker locus, or, in a few cases, large differences in height, coloring, or other morphological features. In 12 remaining cases of doubtful zygosity, four more genetic markers were typed (haptoglobin, phosphoglucomutase, group- 
specific component, and $\mathrm{Xg}^{\mathrm{a}}$ ). It is possible, however, that there are a few pairs diagnosed as monozygotic (MZ) who on still further typing would prove to be DZ.

To maximize the power of the genetic analysis an attempt was made to test equal numbers of the five sex-zygosity classes of twin pairs. Of the 206 twin pairs successfully tested there were $43 \mathrm{MZ}$ female, $42 \mathrm{MZ}$ male, $44 \mathrm{DZ}$ female, $38 \mathrm{DZ}$ male, and $39 \mathrm{DZ}$ pairs of opposite sex (DZOS). There were no substantial differences in age distribution among the five zygosity groups.

\section{Experimental Protocol}

All testing was carried out in the laboratories of the Department of Pharmacology, University of Sydney. Each twin participating attended a testing session beginning at about 9:00 $\mathrm{AM}$, having been asked to eat a light, nonfatty breakfast (no butter, margarine, or milk) about an hour earlier. Before any alcohol was ingested, a breath alcohol reading was taken to ensure that subjects were alcohol free. They were then given an alcohol dose of $0.75 \mathrm{~g}$ ethanol $/ \mathrm{kg}$ body weight (May and Baker, ethanol absolute Proanalys analytical reagent) diluted to $10 \%(\mathrm{v} / \mathrm{v})$ in sugarless lemon squash. They drank this under supervision over a 20 -min period at a constant rate. After a further 20 min, repeated measurements of blood alcohol concentration (BAC) were begun.

For twins who participated in the study during 1979 (78 pairs, 26 also on a second occasion) blood alcohol was measured at nine different times spaced at regular intervals over a 5.5 -hr period. For the remaining 128 pairs (15 also on a second occasion) tested in 1980-1981, blood alcohol was measured at six different times regularly spaced over a 3.5 -hr period. [The schedule was changed after 1979 because preliminary analysis of psychomotor performance (Martin et al., 1981) had shown little effect of alcohol beyond $3 \mathrm{hr}$ after ingestion.] Although no duplicate readings were taken during 1979, virtually all blood alcohol readings were duplicated in $1980-1981$.

\section{Blood Alcohol Measurements}

Finger pads were swabbed with isopropanol and then dried with an electric hair drier. Finger pricks were made with a lancet, either manually or with an automatic device (Autolet, O. Mumford Ltd, U.K.). Whenever possible, a different finger was used for each blood sample. Blood was taken into a $0.25-\mathrm{ml}$, heparinized capillary tube. An $0.1-\mathrm{ml}$ aliquot was pipetted from each tube into a $1-\mathrm{ml}$ reaction vial containing $0.9 \mathrm{ml}$ of 
$0.01 \%(\mathrm{v} / \mathrm{v}) n$-propanol used as the internal standard. The $n$-propanol solution also contained $0.1 \mathrm{~g} /$ /iter mercuric chloride as a preservative. Vials were sealed with caps containing a nonreactive rubber septum and shaken to ensure hemolysis. The duplicate assays taken in 1980-1981 were based on different $0.1-\mathrm{ml}$ aliquots of the original samples.

Most samples were assayed for ethanol concentration within $24 \mathrm{hr}$, and if not, they were frozen at $-20^{\circ} \mathrm{C}$ until subsequent analysis, usually within a week. There was no evidence, from independent tests, that BACs altered after freezing for this period of time.

Samples were analyzed by gas-liquid chromatography using a Hewlett Packard 5700 instrument with a $1.5-\mathrm{m}$ stainless-steel helix $(3.2-\mathrm{mm}$ diameter) packed with $10 \%$ Carbowax 600 (Supelco, Inc.). The operating conditions were as follows: column, $75^{\circ} \mathrm{C}$; detector, $250^{\circ} \mathrm{C}$; and injection port, $250^{\circ} \mathrm{C}$. The gas flows were as follows: nitrogen carrier, $60 \mathrm{ml} / \mathrm{min}$; air, $240 \mathrm{ml} / \mathrm{min}$; and hydrogen, $40 \mathrm{ml} / \mathrm{min}$.

On each day of testing a standard curve was calculated using duplicate samples of known ethanol concentrations $(0,25,50,75$, and $100 \mathrm{mg}$ ethanol $/ 100 \mathrm{ml}$ ) prepared in exactly the same way as blood samples. During the day additional standard samples $(50 \mathrm{mg} / 100 \mathrm{ml})$ were interspersed with blood samples to check for instrument drift. Standard curves were calculated using the ethanol:propanol ratio.

\section{Parameters of the BAC Profile}

Our raw data present a number of problems for subsequent analysis. First, there were differences in the sampling schedule employed in collection of the 1979 and 1980-1981 data. Second, although most blood samples were taken within a couple of minutes of the prescribed time, occasionally, due to problems with the subjects or equipment, they were delayed by as much as 10 or even $15 \mathrm{~min}$, and some samplings were missed altogether. The numbers of such missing observations are apparent from Table I.

Despite these heterogeneities, we were able to obtain standardized data for each individual by fitting to the raw observations a curve of the form

$$
B(t)=A_{0}\left(1-e^{-k_{1} t}\right)+k_{2} t
$$

using a nonlinear least-squares algorithm (Numerical Algorithms Library Mark 7, routine E04FDF). Since there were at least three BACs (and the times at which they were taken) recorded for every individual, least- 
squares estimates of the unknown parameters $A_{0}, k_{1}$, and $k_{2}$ could then be obtained and these could be used to predict the BAC of the individual at any time $t, B(t)$. Separate curves were fitted for each individual. Where duplicate readings were available (in 1980-1981), the mean of duplicates was counted as a single observation. Where an individual attended on two occasions, separate curves were fitted for each occasion.

In the equation above, $A_{0}$ is the notional concentration of alcohol if the dose were instantly ingested and spread throughout the body at time zero. The variables $k_{1}$ and $k_{2}$ are related to the rates of absorption and elimination, respectively. The equation implies that the absorption phase is of a negative exponential form, while the elimination phase is essentially linear. The three estimated parameters are highly intercorrelated and are not readily interpretable, so we shall consider for genetical analysis three transformations of them which are more familiar in the literature of alcohol metabolism.

The predicted time to peak BAC is obtained by differentiating the expression with respect to $t$ and equating this to zero,

$$
\frac{d B(t)}{d t}=A_{0} k_{1} e^{-k_{1} t}-k_{2}=0
$$

so

$$
t_{\max }=\frac{1}{k_{1}} \ln \left(k_{1} A_{0} / k_{2}\right) .
$$

The peak $B A C$ is then predicted as $B\left(t_{\max }\right)$. We also calculated a rate of alcohol elimination as

$$
\left[\hat{B}(3 \mathrm{hr})-\hat{B}\left(t_{\max }\right)\right] /\left(3 \mathrm{hr}-t_{\max }\right) .
$$

For certain regression and correlation analyses in which BACs are employed, values were predicted for each individual at the six mean observation times for blood. In the individuals tested in 1979, up to nine observed blood readings taken from 50 to $300 \mathrm{~min}$ have been used to predict six readings from 56 to $182 \mathrm{~min}$. The means and standard deviations of observed and predicted alcohol concentrations and the parameters derived from them are shown in Table I. Comparison of the degrees of freedom attached to corresponding observed and predicted values shows that although a few degrees of freedom were "manufactured" to contribute to the predicted values (due to interpolation of missing data, etc.), the extra observations collected in 1979 beyond $182 \mathrm{~min}$ used in predicting values at the specified times brought about a net reduction in the degrees of freedom overall. 


\section{RESULTS}

\section{Error of Duplicate Measurements}

Duplicate BAC assays were made on 1631 blood samples (mean BAC, $84 \mathrm{mg} / 100 \mathrm{ml}$ ) during $1980-1981$. From an analysis of variance the mean square between samples $\left(M S_{\mathrm{S}}\right)$ was 442.5 and that between duplicates within samples $\left(M S_{\mathrm{D}}\right)$ was 7.7 . The repeatability, or intraclass correlation

$$
\left[\text { calculated as }\left(M S_{\mathrm{S}}-M S_{\mathrm{D}}\right) /\left(M S_{\mathrm{S}}+M S_{\mathrm{D}}\right)\right] \text {, }
$$

$r_{i}=0.966$ so only $3.4 \%$ of the total variation in blood samples could be ascribed to aliquotting or measurement error. The error of a single BAC measurement was $\sqrt{M S_{\mathrm{D}}}=2.8$, so the $95 \%$ confidence intervals of a single blood alcohol assay were $\pm 5.4 \mathrm{mg} / 100 \mathrm{ml}$.

To check whether measurement errors were uniform over the whole range of BAC values, the absolute differences between duplicate measures were correlated with their sums. The correlation $(r=0.037)$ was trivial and nonsignificant, indicating no heteroscedasticity.

\section{Means and Variances}

The means and standard deviations of observed and predicted blood alcohol concentrations and the parameters derived from them are shown in Table I. Predicted values are also shown graphically in Fig. 1. Correlations between observed and predicted BACs at all sampling times were greater than 0.92. Variances of predicted BACs were lower than those of the corresponding observed readings, but this was expected given that deviations from the prescribed sampling times were eliminated in the predicted values. Female BACs were higher than those for males throughout the range. Variances were higher at earlier than later sampling times but male and female variances were not significantly different.

\section{Repeatability of Measurements Between Testing Occasions}

Analyses of variance of $\mathrm{BAC}$ readings on the individuals who successfully completed the protocol on two occasions were carried out. Repeatabilities $\left[\left(M S_{\mathrm{b}}-M S_{\mathrm{w}}\right) /\left(M S_{\mathrm{b}}+M S_{\mathrm{w}}\right)\right.$, where $M S_{\mathrm{b}}$ is the mean square between individuals and $M S_{\mathrm{w}}$ is the mean square within individuals on the two occasions] were calculated for observed and predicted values (Table II). Repeatabilities for individual sampling times were generally higher for predicted than observed BACs, so demonstrating the efficacy of interpolation to remove error due to heterogeneity of sampling times. 


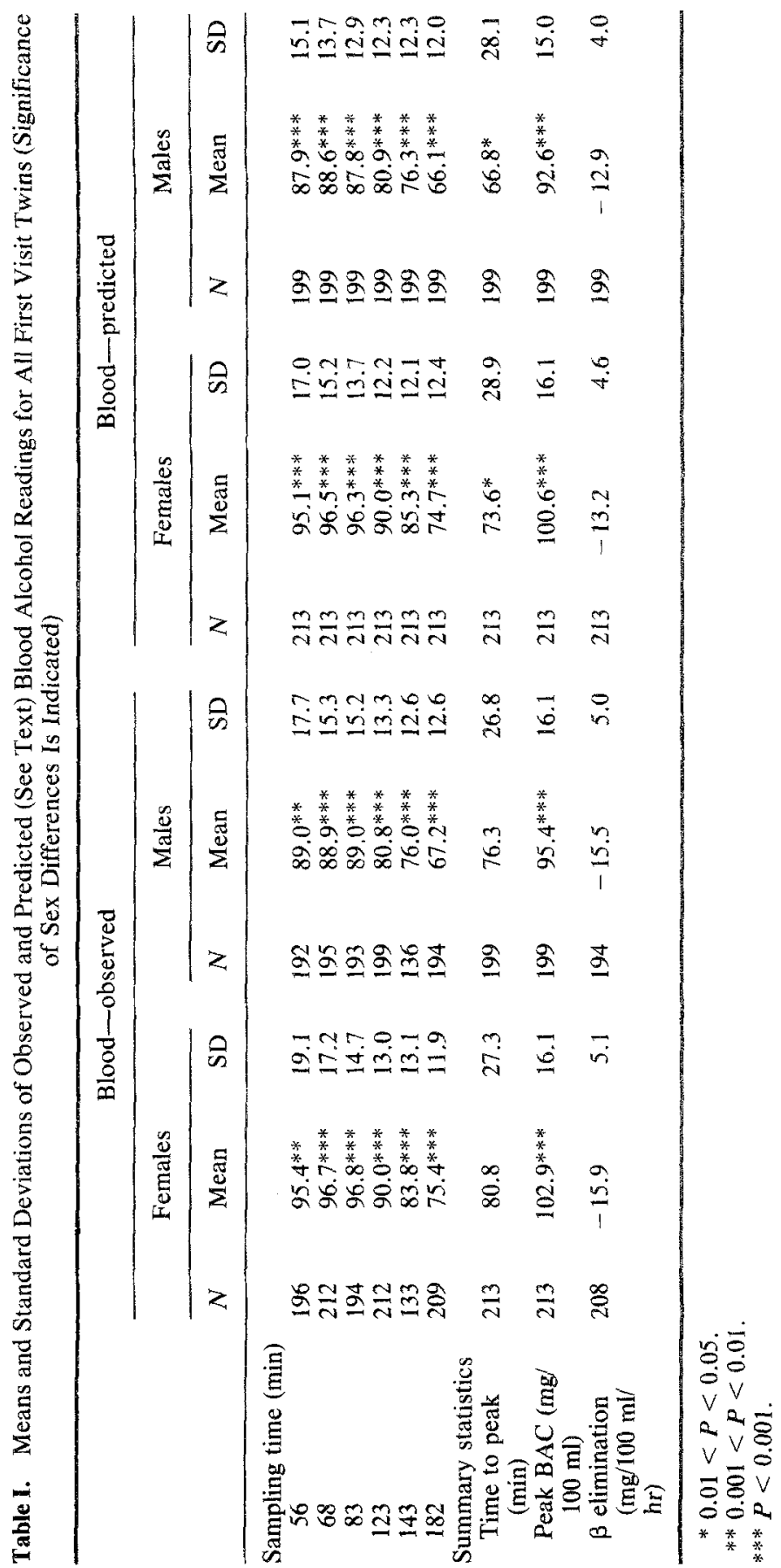




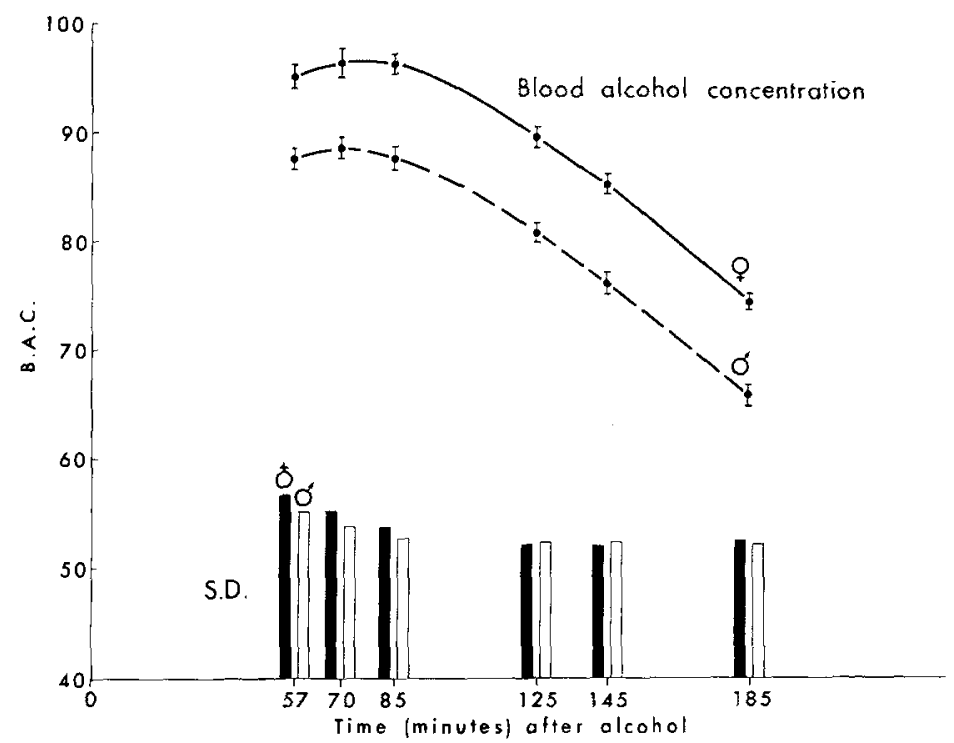

Fig. 1. Time course of blood alcohol concentrations (predicted values as $\mathrm{mg} / 100 \mathrm{ml}$ ) after the ingestion of $0.75 \mathrm{~g} / \mathrm{kg}$ ethanol from 0 to $20 \mathrm{~min}$. Standard error bars are given around the mean values and standard deviations are represented on the same scale along the baseline.

Table II. Repeatabilities for Observed $\left(r_{\mathrm{o}}\right)$ and Predicted $\left(r_{\mathrm{p}}\right)$ Blood Alcohol Concentrations

\begin{tabular}{lcccc}
\hline & $N$ & $r_{\mathrm{o}}$ & $N$ & $r_{\mathbf{p}}$ \\
\hline Sampling time (min) & & & & \\
56 & 69 & $0.52^{* *}$ & 80 & $0.67^{* *}$ \\
68 & 77 & $0.64^{* *}$ & 80 & $0.70^{* *}$ \\
83 & 68 & $0.43^{* *}$ & 80 & $0.69^{* *}$ \\
123 & 79 & $0.63^{* *}$ & 80 & $0.61^{* *}$ \\
143 & 30 & $0.42^{* *}$ & 80 & $0.59^{* *}$ \\
182 & 77 & $0.48^{* *}$ & 80 & $0.57^{* *}$ \\
Mean & & $0.52)$ & & $(0.64)$ \\
Summary statistics & & & & \\
Time to peak & 80 & 0.12 & 80 & $0.27^{*}$ \\
Peak BAC & 80 & $0.66^{* *}$ & 80 & $0.66^{* *}$ \\
$\beta$ elimination & 79 & $0.42^{* *}$ & 80 & $0.39^{* *}$ \\
\hline
\end{tabular}

$* 0.001<P<0.01$.

** $P<0.001$. 
Table III. Correlations of Breakfast, Last Drink, Log Years Drinking, and Log Weekly Alcohol Consumption with Predicted Blood Alcohol Readings $(N=213$ in Females and 199 in Males)

\begin{tabular}{|c|c|c|c|c|c|c|}
\hline \multirow[b]{2}{*}{ Variable } & \multicolumn{6}{|c|}{ Sampling time } \\
\hline & 1 & 2 & 3 & 4 & 5 & 6 \\
\hline \multicolumn{7}{|l|}{ Breakfast } \\
\hline Females & 0.10 & 0.08 & 0.05 & 0.02 & 0.02 & 0.02 \\
\hline Males & -0.02 & 0.01 & 0.03 & 0.07 & 0.09 & 0.10 \\
\hline \multicolumn{7}{|l|}{ Last Drink } \\
\hline Females & 0.06 & 0.05 & 0.02 & -0.05 & -0.09 & $-0.16^{*}$ \\
\hline Males & $0.21^{* *}$ & $0.19^{* *}$ & $0.16^{*}$ & 0.06 & 0.02 & -0.05 \\
\hline \multicolumn{7}{|c|}{ Log years drinking } \\
\hline Females & $0.24^{* *}$ & $0.24^{* * *}$ & $0.24^{* * *}$ & $0.21^{* *}$ & $0.19^{* *}$ & $0.14^{*}$ \\
\hline Males & $0.33^{* * *}$ & $0.32 * * *$ & $0.31^{* * *}$ & $0.25^{* * *}$ & $0.21^{* *}$ & $0.16^{*}$ \\
\hline \multicolumn{7}{|c|}{$\begin{array}{l}\text { Log weekly alcohol } \\
\text { consumption }\end{array}$} \\
\hline Females & $0.26^{* * *}$ & $0.26^{* * *}$ & $0.26^{* * *}$ & $0.21 * *$ & $0.17^{*}$ & 0.10 \\
\hline Males & $0.16^{*}$ & $0.15^{*}$ & 0.13 & 0.07 & 0.04 & -0.02 \\
\hline
\end{tabular}

The average repeatability over all predicted sampling times was 0.64 , that for peak BAC was 0.66 , that for time to peak was 0.27 , and that for rate of elimination was 0.39 . These imply considerable variation in BAC profiles within individuals between testing occasions. Since the correlation between duplicate assays of the same sample was 0.97 , little of this nonrepeatable variation can be attributed to errors of measurement in aliquotting or machine fluctuations.

\section{Correlation of BACs with Drinking Habits and Size of Breakfast}

We examined the effects on BAC measurements of four variables relating to the recent ingestion of alcohol and food. Breakfast on the morning of the test was scored as $\mathbf{0}$ for no breakfast at all, 1 for liquids only, and 2 for liquids plus light (nonfatty) solids. Last drink examined the effects of alcohol drunk on the previous evening and was scored dichotomously as 0 for no alcohol and 1 for some alcohol. Information was also obtained on the age at which subjects started drinking alcohol regularly and on their normal alcohol intake. These were scored as Log years drinking and Log weekly consumption (number of drinks).

The correlations between these four variables and the six predicted blood alcohol readings are shown in Table III. Breakfast was not signif- 
icantly correlated with any of the six BAC readings in either sex. This was expected, as subjects had been asked beforehand not to eat butter, cheese, milk, or anything else fatty for breakfast on the day of the test. Four of the twelve correlations between last drink and BAC were significant, and there was a trend in both sexes for the correlations of last drink and $\mathrm{BAC}$ to become more negative with time. Thus the consumption of alcohol on the previous evening was associated with higher initial BACs but lower readings later in the curve.

Log years drinking was significantly positively correlated with almost all the BAC readings in both sexes, and log weekly alcohol consumption was significantly positively correlated with almost all the $\mathrm{BAC}$ readings in females. Although smaller than in females, the correlations between $\log$ weekly alcohol consumption and BAC in males were again generally positive. The correlations of both log years drinking and log weekly alcohol consumption with BACs were lowered only slightly in either sex by partialing out the effects of age. Within the age group 18-34 years, then, habits of heavier drinking were associated with higher readings after the standard dose throughout the BAC profile. The direction of causation in this association could not, of course, be inferred. While prolonged drinking habits may lead to higher BACs after a standard dose (due to deterioration in liver function), it is also possible that individuals who reach higher BACs find drinking more pleasurable, which reinforces the habit.

\section{Correction of BACs for Effects of Sex, Age, and Physical Measures}

Here we examine the effects on BAC measures of sex, age, and some physical measurements which might have accounted for the repeatable, perhaps genetic, proportion of $\mathrm{BAC}$ variances. The five physical measures included in the preliminary analyses were height, ponderal index (height/ $\sqrt[3]{\text { weight }})$, adiposity $\{[\log (x-18)]$, where $x$ is the subscapular skinfold thickness measured in $0.1 \mathrm{~mm}$, a measure of lung function (FVC, forced vital capacity), and weight (despite the fact that there had already been one allowance for weight in the dose administered). A second measure of lung function (FEV, forced expiratory volume) was not included because it was very highly correlated with FVC.

Initially, several two-way analyses of covariance were carried out in which the two cross-classified factors were the 402 individuals for whom all measurements were available and the six sampling times. The covariates were age, the various physical measurements, and sex (which could also be regarded as a third factor with two levels). 
Table IV. Correlations of Predicted Blood Alcohol Readings with Age and Physical Measures $(N=207$ in Females and 195 in Males)

\begin{tabular}{|c|c|c|c|c|c|c|}
\hline \multirow[b]{2}{*}{ Variable } & \multicolumn{6}{|c|}{ Sampling time } \\
\hline & 1 & 2 & 3 & 4 & 5 & 6 \\
\hline \multicolumn{7}{|l|}{ Age } \\
\hline Females & $0.18^{*}$ & $0.15^{*}$ & 0.12 & 0.03 & -0.00 & -0.05 \\
\hline Males & $0.29^{* * *}$ & $0.31^{* * *}$ & $0.32^{* * *}$ & $0.31^{* * *}$ & $0.29^{* * *}$ & $0.26^{* * *}$ \\
\hline \multicolumn{7}{|l|}{ Weight } \\
\hline Females & $0.15^{*}$ & $0.21^{* *}$ & $0.27 * * *$ & $0.39 * * *$ & $0.42 * * *$ & $0.45^{* * * *}$ \\
\hline Males & $0.20^{* *}$ & $0.25 * * *$ & $0.30 * * *$ & $0.35 * * *$ & $0.35^{* * *}$ & $0.34 * * *$ \\
\hline \multicolumn{7}{|l|}{ Adiposity } \\
\hline Females & $0.25^{* * *}$ & $0.29 * * *$ & $0.32^{* * * *}$ & $0.35^{* * *}$ & $0.35^{* * *}$ & $0.33^{* * *}$ \\
\hline Males & $0.16^{*}$ & $0.21 * *$ & $0.25 * * *$ & $0.31^{* * *}$ & $0.32 * * *$ & $0.32^{* * *}$ \\
\hline \multicolumn{7}{|c|}{ Lung function (FVC) } \\
\hline Females & $-0.15^{*}$ & $-0.14^{*}$ & -0.14 & -0.11 & -0.10 & -0.08 \\
\hline Males & $-0.16^{*}$ & $-0.17^{*}$ & $-0.17^{*}$ & $-0.15^{*}$ & $-0.15^{*}$ & -0.14 \\
\hline
\end{tabular}

$* 0.01<P<0.05$.

$* * 0.001<P<0.01$.

*** $P<0.001$.

After fitting the covariates in stepwise fashion in many combinations, it was found that any effect of height, (height $)^{2}$, or $(1 / \text { height })^{2}$ could be better accounted for by FVC. Similarly, there was no effect of the ponderal index beyond that observed in the effects of weight and adiposity. Therefore, the only four covariates included in the final analyses apart from sex were age, weight, adiposity, and FVC. Raw correlations of the six predicted blood BACs with these four covariates are shown in Table IV. The estimates of all parameters included in the final analysis of covariance model are shown in Table $\mathrm{V}$. The grand mean in Table $\mathrm{V}$ is that for females at Time 1, and the main effects for sex, time, and the covariates are represented as deviations from the grand mean. Inspection of the significant estimates for the main effects suggests that higher BACs were associated with female sex, greater age, weight, and skin-fold thickness, and lower lung volume.

However, inspection of the correlations in Table IV and of the three significant types of two-way interactions in Table $\mathrm{V}$ indicates a more complex set of relationships. The three significant types of interaction all involve sampling time, and the estimates for specific interaction effects in Table $\mathrm{V}$ are each obtained as the cumulative deviation from the grand mean of the particular main effect and its interaction term with sampling time. It is apparent that at later stages of the blood ethanol curve the effect of age decreases, while the effect of weight and the sex difference increase. 
Table V. Parameter Estimates with Their Standard Errors and Significance Levels for the Final Linear Model Predicting Blood Alcohol Levels in Terms of Sex, Time, Age, Adiposity, Weight, Lung Function, and Necessary Interaction Terms (Grand Mean Is That Predicted for Females at Time 1)

\begin{tabular}{lccccc}
\hline \multicolumn{1}{c}{ Main effects } & Estimate & SE & $\begin{array}{c}\text { Two-way } \\
\text { interactions }\end{array}$ & Estimate & SE \\
\hline Grand mean & $71.15^{* * *}$ & 4.92 & Age $\cdot$ time (2) & -0.10 & 0.17 \\
Male & $-4.07^{* *}$ & $\mathbf{1 . 5 0}$ & Age $\cdot$ time (3) & -0.21 & 0.17 \\
Time (2) & 0.36 & 6.22 & Age $\cdot$ time (4) & $-0.39^{*}$ & 0.17 \\
Time (3) & -0.94 & 6.22 & Age $\cdot$ time (5) & $-0.46^{* *}$ & 0.17 \\
Time (4) & -8.75 & 6.22 & Age $\cdot$ time (6) & $-0.57^{* * *}$ & 0.17 \\
Time (5) & $-13.16^{* * *}$ & 6.22 & Male $\cdot$ time (2) & -1.49 & 1.98 \\
Time (6) & $-22.97^{* * *}$ & 6.22 & Male $\cdot$ time (3) & -2.82 & 1.98 \\
Age & $0.57^{* * *}$ & $\mathbf{0 . 1 2}$ & Male $\cdot$ time (4) & $-4.42^{*}$ & 1.98 \\
Adiposity & $0.03^{* * *}$ & 0.01 & Male $\cdot$ time (5) & $-4.64^{*}$ & 1.98 \\
Weight & $0.29^{* * *}$ & 0.07 & Male $\cdot$ time (6) & $-4.55^{*}$ & 1.98 \\
Lung function (FVC) & $-4.60^{* * *}$ & 0.38 & Weight $\cdot$ time (2) & 0.06 & 0.10 \\
& & & Weight $\cdot$ time (3) & 0.12 & 0.10 \\
& & & Weight $\cdot$ time (4) & $0.22^{*}$ & 0.10 \\
& & & Weight $\cdot$ time (5) & $0.24^{*}$ & 0.10 \\
& & & Weight $\cdot$ time (6) & $0.27^{* *}$ & 0.10 \\
\hline
\end{tabular}

\footnotetext{
$* 0.01<P<0.05$.

$* * 0.001<P<0.01$.

*** $P<0.001$.
}

In fact it is clear from the correlations with age in Table IV that there was also at least one important three-way interaction; the correlations between age and BAC values declined with sampling times in females but not males. Unfortunately, limitations of available computer storage precluded the fitting of models with three-way interaction terms.

Nevertheless, it is clear from the correlations and two-way interactions that different factors influenced BACs during the absorption and elimination phases. While adiposity and FVC were equally important in both phases, sex and weight differences were more important during elimination than absorption, and the effects of age, at least in females, were confined mainly to the absorption phase (age differences in males being of similar importance in both phases). Whatever the changes in their individual effects over time, however, multiple regression analysis indicated that the combined effects of age, weight, adiposity, and lung volume could account for no more than $10 \%$ of the total BAC variance at any given time in either age.

Finally, three perhaps surprising features of the results in Tables IV and $\mathrm{V}$ deserve further comment. First, the positive association with adiposity was expected because alcohol would be disproportionately con- 
Table V1. Observed Mean Squares for Parameters of Blood Alcohol Metabolism

\begin{tabular}{|c|c|c|c|c|}
\hline & $\mathrm{df}$ & Time to peak & Peak BAC & $\begin{array}{l}\text { Rate of elimination } \\
\qquad\left(\times 10^{6}\right)\end{array}$ \\
\hline \multicolumn{5}{|l|}{ MZ females } \\
\hline Between & 42 & 1038 & 371 & 27.12 \\
\hline Within & 43 & 353 & 89 & 8.56 \\
\hline \multicolumn{5}{|l|}{$\mathrm{MZ}$ males } \\
\hline Between & 41 & 1471 & 361 & 27.08 \\
\hline Within & 42 & 496 & 92 & 9.10 \\
\hline \multicolumn{5}{|l|}{ DZ females } \\
\hline Between & 43 & 1316 & 294 & 31.72 \\
\hline Within & 44 & 440 & 226 & 18.44 \\
\hline \multicolumn{5}{|l|}{ DZ males } \\
\hline Between & 37 & 959 & 336 & 15.28 \\
\hline Within & 38 & 448 & 131 & 12.17 \\
\hline \multicolumn{5}{|c|}{ DZ opposite sex } \\
\hline Between & 38 & 735 & 373 & 20.76 \\
\hline Within $^{a}$ & 38 & 948 & 170 & 16.17 \\
\hline
\end{tabular}

${ }^{a}$ Corrected for mean sex difference.

centrated in nonadipose tissue. However, the additional positive association with weight probably indicates that the measure of subscapular skin-fold thickness was an imperfect indicator of total body fat. Second, the significant negative correlations of BAC and FVC suggested that subjects with a greater lung capacity may have been able to clear more alcohol through the lungs. Third and perhaps most important, none of the covariates was able to account for the large sex differences in BACs. No matter which of the covariates was entered into the regression equation, the model could always be improved by about the same amount when sex was added as a factor in the model.

\section{Genetic Analysis}

Analyses of variance were carried out between and within pairs of twins for each of the five twin groups. The resultant mean squares are shown in Table VI for the three summary statistics of the blood alcohol curve, viz., peak BAC, time to peak, and rate of elimination. Alternative models of variation are fitted to the observed mean squares, and their goodness of fit is tested by the chi-square criterion. Possible sources of variation to be included in the models are $E_{1}$, individual environmental variation (including measurement error), $E_{2}$, which is environmental variation due to influences shared by cotwins but differing between families, and $V_{\mathrm{A}}$, which is additive genetic variation. The expectations of the mean 
Table VII. Results of Fitting Models to Mean Squares for Peak BAC and Rate of Elimination

\begin{tabular}{lccrrrrr}
\hline Model & $E_{1}$ & $E_{2}$ & $V_{\mathrm{A}}$ & $\mathrm{df}$ & $\chi^{2}$ & $P$ & $h^{2}$ \\
\hline \multicolumn{7}{c}{ Peak BAC } \\
$E_{1}$ & 243 & & & 9 & 42.44 & 0.00 & \\
$E_{1} E_{2}$ & 142 & 102 & & 8 & 15.01 & 0.06 & \\
$E_{1} V_{\mathrm{A}}$ & 93 & & 153 & 8 & 4.40 & 0.82 & $0.62 \pm 0.06$ \\
$E_{1} E_{2} V_{\mathrm{A}}$ & 92 & -10 & 164 & 7 & 4.21 & 0.75 & \\
& & & Rate of elimination & & & \\
$E_{1}$ & 18.73 & & & 9 & 34.05 & 0.00 & \\
$E_{1} E_{2}$ & 12.87 & 5.92 & & 8 & 14.93 & 0.06 & \\
$E_{1} V_{\mathrm{A}}$ & 9.51 & & 9.42 & 8 & 8.13 & 0.42 & $0.49 \pm 0.07$ \\
$E_{1} E_{2} V_{\mathrm{A}}$ & 8.98 & -2.96 & 12.84 & 7 & 7.66 & 0.36 & \\
\hline
\end{tabular}

squares in terms of these parameters and the weighted least-squares procedure by which models are fitted are described extensively elsewhere (e.g., Eaves et al., 1978).

Results of fitting models to the mean squares for peak $\mathrm{BAC}$ and rate of elimination are shown in Table VII. There was no significant heterogeneity of fit over sexes so the fit of models to all 10 mean squares is shown. For both variables a model specifying that all variation is due to individual environmental differences and error variation $\left(E_{1}\right)$ is strongly rejected. A model which also allows for shared environment but not for genetic variation $\left(E_{1} E_{2}\right)$ is not quite rejected at the $5 \%$ significance level but, nevertheless, gives a poor fit for both variables. The model which specifies only individual environment and additive genetic variance $\left(E_{1} V_{\mathrm{A}}\right)$, on the other hand, gives a good fit to the data, and no significant improvement is obtained for either variable by adding $E_{2}$ to the model.

Heritabilities are estimated as $0.62 \pm 0.06$ for peak $\mathrm{BAC}$ and 0.50 \pm 0.07 for rate of elimination. These estimates do not differ significantly from the repeatabilities, $0.66 \pm 0.11$ and $0.39 \pm 0.11$, respectively (Table II).

Results for time to peak are not so clear. While a genetic model is not inconsistent with the same-sex twin data, shared environment is a more likely explanation of the familial aggregation for this variable. In the opposite-sex pairs there is not even any evidence of familial aggregation. The inability to detect, with any degree of confidence, genetic variation for time to peak is consistent with the very low repeatability $(0.27)$ of this variable. 


\section{DISCUSSION}

This study has revealed considerable variation between individuals in all aspects of their blood ethanol profiles following the ingestion of a weight-related dose of ethanol. About two-thirds of this variance between individuals in peak BAC, one-half in rate of elimination, and less than one-third in time to peak was found to be repeatable between occasions. Results of fitting models to twin mean squares were consistent with the conclusion that all the repeatable variance in peak BAC and rate of elimination is genetic in origin. However, only a small fraction of the nonrepeatable variance between occasions could be attributed to measurement errors. We conclude that relatively short-term environmental factors have considerable influence on blood alcohol metabolism.

A significant but still small $(<12 \%)$ fraction of variance was due to variation in drinking experience. In the short term, consumption of alcohol during the evening prior to the test was associated with higher BAC readings during absorption but lower readings during elimination (even though all twins had zero BAC readings immediately before they began the test). In the longer term, the total number of years drinking and the average weekly consumption of alcohol were both positively associated with BACs throughout the profile. However, the direction of causation in these associations with longer-term drinking experience could not be inferred; it is possible either that liver damage associated with prolonged heavy drinking could impair ethanol metabolism or that higher BACs could produce a more pleasurable experience, which could reinforce the drinking habit.

Little contribution to the nonrepeatable variance between occasions was made by the amount of breakfast on the morning of the test. However, this was not surprising because subjects had been asked to exclude fatty foods from this meal. It was also possible that longer-term changes in diet, and in general health, contributed substantially to the nonrepeatable variance.

An attempt was made to explain the repeatable, heritable variation in terms of various physical characteristics. It was found that BAC values were positively correlated with skin-fold thickness and also with weight, even though the dose ingested had been proportional to body weight. The residual correlation with weight suggests that the single measure of skinfold thickness used was not an adequate indicator of overall adiposity. A smaller positive correlation was also found between BAC values and age, but this could be explained either through habit reinforcement or by a decline in general health. A small negative correlation was found between BAC measures and lung volume. Since FVC is a good indicator 
of general physical health (Gibson et al., 1983), this correlation may reflect increased metabolic efficiency in the physically fit.

The regression analysis also showed that females had higher BACs than males. This difference occurred during both phases but was most marked during elimination. This sex difference could not be explained by differences in skin-fold thickness, weight, or lung volume but may be related to differences in the distribution of fatty tissue between the sexes which neither weight nor skin-fold thickness brought out.

The combined contribution of age, weight, adiposity, and lung volume to the total BAC variance was not more than $10 \%$. This was so irrespective of sex and absorption versus elimination. Age per se is not an inherited trait and weight, adiposity, and lung function are incompletely inherited (Clark et al., 1980; Gibson et al., 1983), so the contribution of these four variables to the heritable portion of total $\mathrm{BAC}$ variance must also have been small.

The identity of the most important genetic determinants of BAC values thus awaits further study. However, genetic differences in the activity levels of the enzymes alcohol dehydrogenase and acetaldehyde dehydrogenase have recently been described [Goedde et al. (1979) and references therein]. These enzymes catalyze the first two steps in the catabolism of ingested ethanol to acetyl-CoA and racial comparisons link variation in acetaldehyde dehydrogenase activity in particular to variation in susceptibility to intoxication (Gibson and Oakeshott, 1981; Harada et $a l ., 1982$ ). Now that parameters of the BAC profile are known to be under genetic control, an investigation of the contribution of these inherited enzyme differences to the genetics of the BAC profile would seem well worthwhile.

\section{ACKNOWLEDGMENTS}

We thank the twins for their cooperation, Janet Craig for day-to-day administration, Dr. L. Y. C. Lai for zygosity determination, Mrs. M. Olsen for expert practical assistance, Mr. D. Rowell for assistance with computations, Drs. M. A. Adena and S. R. Wilson for statistical advice, and Dr. L. J. Eaves for helpful discussions.

\section{REFERENCES}

Clark, P., Jardine, R., Martin, N. G., Stark, A. E., and Walsh, R. J. (1980). Sex differences in the inheritance of some anthropometric characters in twins. Acta Genet. Med. Gemellol. 29:171-192.

Eaves, L. J., Last, K. A., Young, P. A., and Martin, N. G. (1978). Model-fitting approaches to the analysis of human behavior. Heredity 41:249-320. 
Gibson, J. B., and Oakeshott, J. G. (1981). Genetics of biochemical and behavioural aspects of alcohol metabolism. Aust. N.Z. J. Med. 11:128-131.

Gibson, J. B., Martin, N. G., Oakeshott, J. G., Rowell, D. M., and Clark, P. (1983). Lung function in an Australian population: Contributions of polygenic factors and the Pi locus to individual differences in lung function in a sample of twins. Ann. Hum. Biol. 10:547556.

Goedde, H. W., Agarwal, D. P., and Harada, S. (1979). Alcohol metabolising enzymes: Studies of isozymes in human biopsies and cultured fibroblasts. Clin. Genet. 16:29-33.

Harada, S., Agarwal, D. P., and Goedde, H. W. (1982). Human aldehyde dehydrogenase: Electrophoretic and biochemical studies of 3,4 dihydroxyphenylacetaldehyde metabolising enzyme and its possible role in alcoholism. Abstract, Titisee Workshop on Alcohol Metabolising Enzymes, January 1982.

Kopun, M., and Propping, P. (1977). The kinetics of ethanol absorption and elimination in twins supplemented by repetitive experiments in single subjects. Eur. J. Clin. Pharmacol. 11:337-344.

Luth, K. F. (1939). Untersuchungen uber die Alkoholblutconzentration nach Alkoholgaben bei 10 eineiigen und 10 zweieigen Zwillingspaaren. Dtsch. Z. Gerichtl. Med. 32:145164.

Martin, N. G., Eaves, L. J., Kearsey, M. J., and Davies, P. (1978). The power of the classical twin study. Heredity 40:97-116.

Martin, N. G., Gibson, J. B., Oakeshott, J. G., Wilks, A. V., Starmer, G. A., Craig, J., and Perl, J. (1981). A twin study of psychomotor performance during alcohol intoxication: Early results. Twin Res. 3C:89-96.

Martin, N. G., Oakeshott, J. G., Gibson, J. B., Starmer, G. A., Perl, J., and Wilks, A. V. (1985). A twin study of psychomotor and physiological response to an acute dose of alcohol. Behav. Genet. (in press).

Vesell, E. S. (1972). Ethanol metabolism: Regulation by genetic factors in normal volunteers under a controlled environment and the effect of chronic ethanol administration. Ann. N.Y. Acad. Sci. 197:79-88.

Wagner, J. G. (1973). Intrasubject variation in elimination halflives of drugs which are appreciably metabolised. J. Pharmacokin. Biopharm. 1:165-173.

Wagner, J. G., and Patel, J. A. (1972). Variations in absorption and elimination rates of ethyl alcohol in a single subject. Res. Comm. Chem. Pathol. Pharmacol. 4:61-76.

Edited by C. R. Cloninger 